\title{
Refuerzo de un edificio mediante la incorporación de un sistema de disipación de energía
}

\section{Building retrofit by incorporating an energy dissipation system}

Presentación: 6-7/10/2020

\section{Doctorando:}

\section{Cristian Otto Bay}

Grupo de Estudio de Estructuras Civiles, Facultad Regional San Rafael, Universidad Tecnológica Nacional - Argentina cbay@frsr.utn.edu.ar

\section{Director/a:}

\section{Gustavo Luis Palazzo}

\section{Co-director/a:}

\section{José Luis Almazán Campillay}

\section{Resumen}

En zonas de alta peligrosidad sísmica existen estructuras que pueden requerir un refuerzo estructural para mejorar su desempeño. Una alternativa para realizar estos refuerzos es la incorporación de sistemas pasivos de energía. Este trabajo tiene por objeto aplicar una metodología de diseño propuesta por el autor a un caso de estudio. Se diseñan los dispositivos y posteriormente se contrasta el método propuesto con análisis dinámico no lineal según requerimientos de la norma americana ASCE/SEI 7-16. Para la excitación sísmica se eligen dos grupos de siete registros representativos de la zona de emplazamiento escalados por ajuste espectral. En base a la consideración de varios parámetros de respuesta se muestra la eficiencia del procedimiento de diseño propuesto.

Palabras clave: Disipadores, Diseño por Desplazamientos, Refuerzo de estructuras

\begin{abstract}
In areas of high seismic hazard, there are structures that may require structural reinforcement to improve their performance. An alternative to carry out these reinforcements is the incorporation of passive energy systems. The purpose of this work is to apply a design methodology proposed by the author to a case study. The devices are designed and subsequently the proposed method is contrasted with non-linear dynamic analysis according to the requirements of the American standard ASCE / SEI 7-16. For seismic excitation, two groups of seven records representative of the site area are chosen scaled by spectral adjustment. Based on the consideration of several response parameters, the efficiency of the proposed design procedure is shown.
\end{abstract}

Keywords: Buckling restrained brace, Displacement Seismic Design, Retroffit, 


\section{Introducción}

Un problema vigente en ingeniería sísmica es la vulnerabilidad de edificios existentes. Esta vulnerabilidad requiere de un refuerzo o rehabilitación estructural que permita a la estructura tener un desempeño adecuado, para lo cual existen diversos procedimientos. Tradicionalmente la incorporación de resistencia y rigidez se ha materializado mediante elementos de hormigón armado, acero, e incluso materiales compuestos. Estas metodologías, en general, son de aplicación compleja y resultan invasivas en lo funcional. Además, implican la paralización de actividades durante largos periodos, que en el caso de edificios esenciales genera problemas de logística importantes (Bay, et al., 2019). Otros métodos están basados en nuevas tecnologías, como los sistemas de aislamiento sísmico y los sistemas de disipación pasiva de energía, los cuales permiten dar solución al refuerzo estructural en menos tiempo que los sistemas convencionales, y también, concentran el daño en estos elementos "fusibles" para luego ser reemplazados (en caso de ser necesario) con posterioridad a un evento sísmico.

Dentro de la práctica actual, se pueden emplear distintas metodologías para el diseño de estructuras con sistemas de disipación: (i) método de diseño basado en fuerzas (combinado con la verificación del objetivo de desplazamiento requerido, Ponzo, et al., 2009); (ii) métodos basados en desplazamientos (donde el diseño comienza a partir de desplazamientos definidos, Kim y Choi, 2004; Bergami y Nutti, 2013; Mazza y Vulcano, 2015; Nuzzo, et al., 2019; Bay, et al., 2019); y (iii) métodos basados en energía (poco difundidos en la práctica profesional).

Es importante destacar que, en las metodologías mencionadas existe una tendencia (ASCE 7, 2016; ASCE 41, 2017) a aceptar preferentemente el análisis dinámico no lineal (ADNL) en el diseño con sistemas de disipación. Como esta clase de análisis requiere un tiempo de cálculo muy alto, resulta necesario contar con métodos de diseño simples y de bajo costo computacional, que permitan estimar las propiedades del sistema de disipación.

En base a la consideración anterior, el objeto de este trabajo es mostrar una aplicación del método propuesto (Bay, et al., 2019) en el refuerzo de un edificio de 6 niveles construido en la década del 70 en zona de elevada peligrosidad sísmica.

En la sección siguiente se presenta un resumen del método de diseño. Luego, se aplica a un pórtico plano de hormigón armado (representativo de un edificio simétrico). Se muestran, además, los parámetros de respuesta (desplazamientos, distorsiones, y demandas de ductilidad) según un ADNL. Para realizar este análisis y establecer los criterios de aceptación se siguen las especificaciones de normas americanas (ASCE 7, 2016; ASCE 41, 2017), considerando acelerogramas escalados al espectro de diseño y al espectro máximo considerado con el procedimiento de ajuste espectral. En la sección final de conclusiones se destacan las ventajas de diseñar el sistema de disipación con el método propuesto basado en desplazamientos para contrastar luego con un ADNL el desempeño requerido para la estructura reforzada

\section{Desarrollo}

El refuerzo estructural se plantea en un edificio en altura de seis niveles en hormigón armado, destinado a oficinas y departamentos, construcción característica de la década del 70 en la ciudad de Mendoza. El edificio ha sido diseñado a cargas verticales dado que a la fecha de su concepción no se contaba con normativa sismorresistente vigente en la provincia. La regularidad del edificio en planta permitió realizar un análisis plano tal como se indica en la figura 1, donde también se indican las cargas y las secciones transversales de los elementos. Se utilizó un hormigón de resistencia $20 \mathrm{MPa}$ y acero de refuerzo ADN420, típicos de Argentina. 

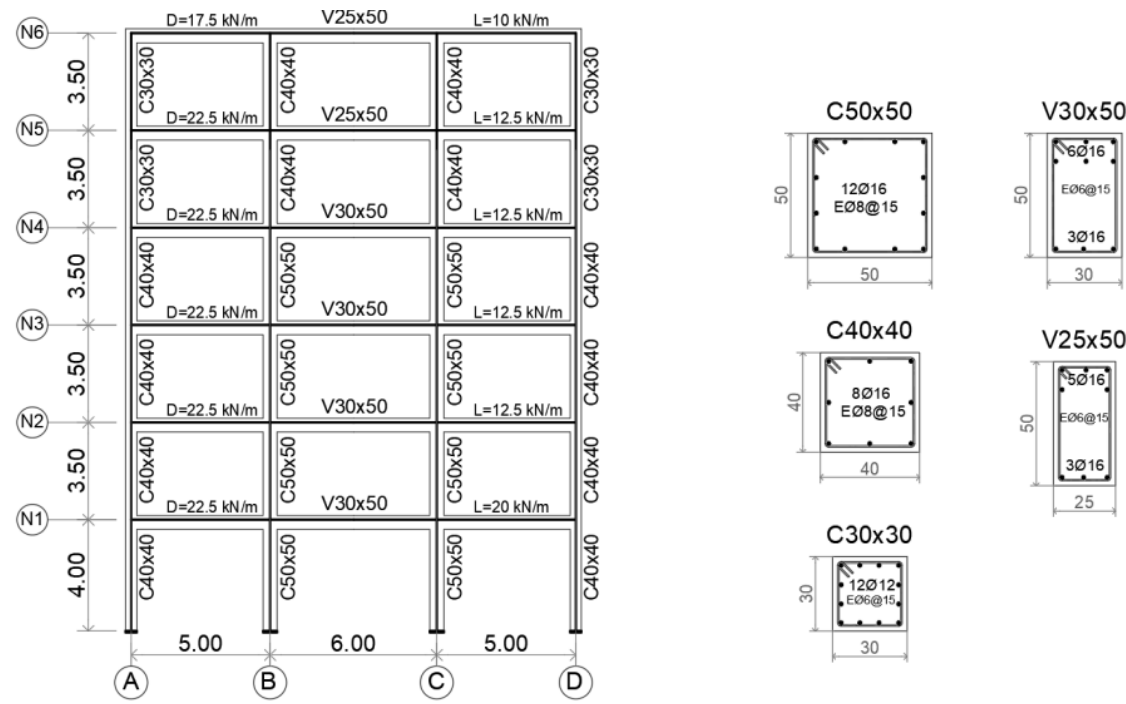

Figura 1: Elevación del pórtico analizado, cargas utilizadas y secciones de hormigón armado.

Un análisis lineal elástico (ETABS 2015) determinó un periodo fundamental de vibración de 1.12 segundos. Resultando una estructura flexible para los requerimientos de normativa nacional (INPRES-CIRSOC 103-I, 2013). Una estimación de la demanda sísmica actual (INPRES-CIRSOC 103-I, 2013), y un análisis estático no lineal (AENL) según normativa estadounidense (ASCE 41, 2017) evidenció la falta de resistencia y rigidez de la estructura para responder ante las demandas sísmicas del sitio.

Para subsanar los problemas de resistencia y rigidez se podría plantear un incremento de secciones de hormigón armado en columnas principalmente, el cual demandaría tiempos importantes de ejecución. Otra alternativa, es adicionar un sistema de disipación de energía que proporcione resistencia y rigidez a la estructura. Entonces, se propone un sistema histerético tipo barras de pandeo restringido (BPR) con el objeto de mejorar el desempeño (figura 2).

El método de diseño propuesto (Bay, et al., 2019) sigue los lineamientos del diseño basado en desplazamientos propuesto originalmente por Priestley, et al. (2007). Como paso preliminar se caracteriza la estructura original mediante un AENL y se transforma en un sistema de un grado de libertad (Fajfar, 2000). Luego se establece un desplazamiento objetivo máximo para el terremoto de diseño (TD) y terremoto máximo considerado (TMC) en función del desempeño esperado. Para el edificio en estudio se adopta un desplazamiento de $11.20 \mathrm{~cm}$ para TD y $22.40 \mathrm{~cm}$ para TMC considerando un nivel de desempeño de ocupación inmediata para TD (FEMA 273). Esto permite determinar una relación de amortiguamiento viscoso equivalente (AVE), donde se recomienda utilizar las expresiones de Yan, etal., (2018). El sistema de disipación se caracteriza por la ductilidad de los disipadores, se considera 10 para este ejemplo, y un factor de endurecimiento posterior a la fluencia, $\mathrm{r}_{\mathrm{SD}}=0.05$. Para el AVE del SD se utiliza la expresión de Liu, et al. (2014). Caracterizados ambos sistemas, se determina el AVE del sistema en paralelo considerando el criterio de que las constantes de amortiguamiento se suman (Bay y Palazzo, 2019). Esto deriva en que el AVE del conjunto depende de los AVE y de los cortes basales de cada sistema. Dado el desconocimiento del corte basal del SD resulta en un método iterativo que con pocos pasos se logra la convergencia. Determinadas las propiedades del SD se distribuyen entre los distintos niveles en forma proporcional al primer modo de vibración (tabla mostrada en la Figura 2). Cabe destacar que en la literatura no se han encontrado métodos de diseño que apliquen este concepto (Mazza y Vulcano, 2008; 2014; Nuzzo, et al., 2019). 


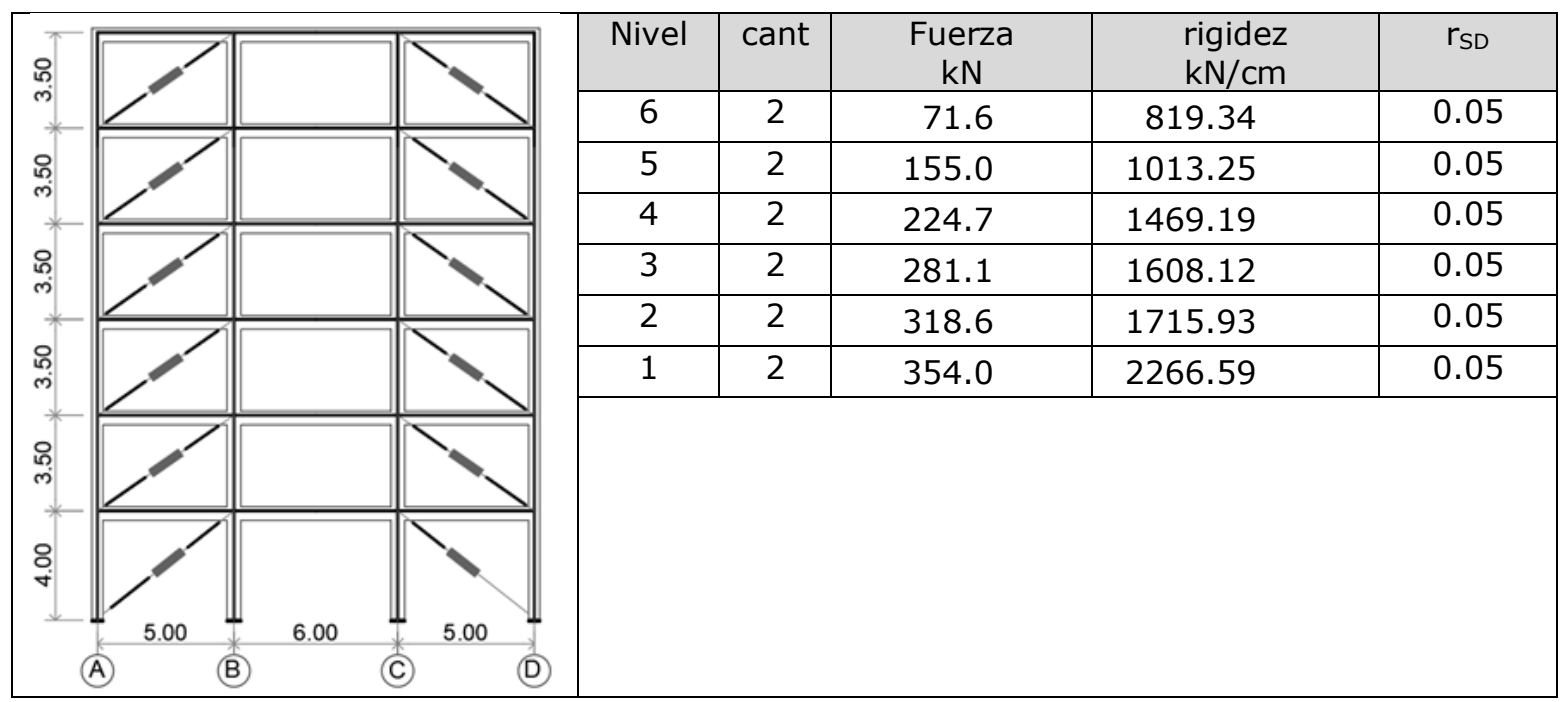

Figura 2: Disposición del sistema de disipación y propiedades de los disipadores

Para evaluar la eficiencia y confiabilidad del método de diseño propuesto se somete a la estructura reforzada a un ADNL. Para la simulación se utiliza un modelo de plasticidad concentrada mediante fibras. En el hormigón se utiliza un modelo de Mander (resistencia característica a compresión, f'c = 20 MPa; y módulo de elasticidad, Ec $=21090 \mathrm{MPa}$ ) y en el acero de refuerzo un modelo bilineal simplificado (Fy = $420 \mathrm{MPa}$; Es = $200000 \mathrm{MPa} ; \mathrm{r}=0.05$ ), para las BPR se utiliza un modelo simplificado bilineal sin degradación de rigidez con $r=0.05$. En el rango elástico se considera un amortiguamiento de Rayleigh proporcional a la rigidez secante de 0.0035 , que aporta un amortiguamiento del $1 \%$.

En el sitio donde se sitúa el edificio se pueden presentar terremotos corticales superficiales, con fallas diversas. Considerando que no se cuenta con mapas de fallas, ni con registros con aceleraciones mayores a 0,52g, se seleccionaron registros de la base de datos del FEMA P695 (2009) que cumplen con una magnitud superior a 6, PGA mayor a $0.30 \mathrm{~g}$ y PGV mayor a $30 \mathrm{~cm} / \mathrm{s}$ (Bay, et al. 2019). se presentan los dos grupos seleccionados, uno de falla lejana y otro de falla cercana no impulsivos, ambos grupos de registros son escalados por ajuste espectral (Abrahamson, N., 1992), según los lineamientos del Capítulo 18 del ASCE-7, (2016).

La respuesta se obtiene aplicando integración directa, a través del método Hilber-Hughes-Taylor (con $\alpha=-0,1, \beta$ $=0,3025$, y $\gamma=0,60$ ). El tiempo de solución para cada simulación fue de entre 10 minutos y 3 horas dependiendo de la no linealidad del modelo, principalmente. Es importante destacar que para todos los registros hubo convergencia.

En la figura 3 se presentan los resultados en máximos promedios de los 14 registros para desplazamientos, distorsiones de piso y demandas de ductilidades de las BPR. En términos de desplazamientos se observa que el SR para TD y TMC mantiene la forma del primer modo, en ambos casos se reducen los desplazamientos respecto del SO, y se obtiene un desplazamiento máximo promedio de $9.60 \mathrm{~cm}$ contra los $11.20 \mathrm{~cm}$ previstos en el diseño. En distorsiones máximas, principal parámetro para evaluar el daño en estructuras, se obtiene una distorsión máxima en el segundo nivel de $0.5 \%$ y 1\% para TD y TMC, valores similares a los estimados en el diseño. Finalmente, en términos de demanda de ductilidad del SD se observa que para TD la demanda resulta de 8.37 y 16.61 para TD y TMC. Estos valores son inferiores a los supuestos en el diseño. No se presentan los resultados de cada uno de los 14 registros, pero el escalamiento por ajuste espectral presenta menores dispersiones en los resultados respecto del clásico ajuste por amplitudes, además requiere menor tiempo computacional para las simulaciones. Otro punto importante a destacar, es que no se ha considerado la excentricidad accidental de la masa, ASCE 7 (2016), en cuyo caso hubiese sido necesario agregar dos casos más de cargas, transformando las 14 simulaciones en 42 simulaciones. 

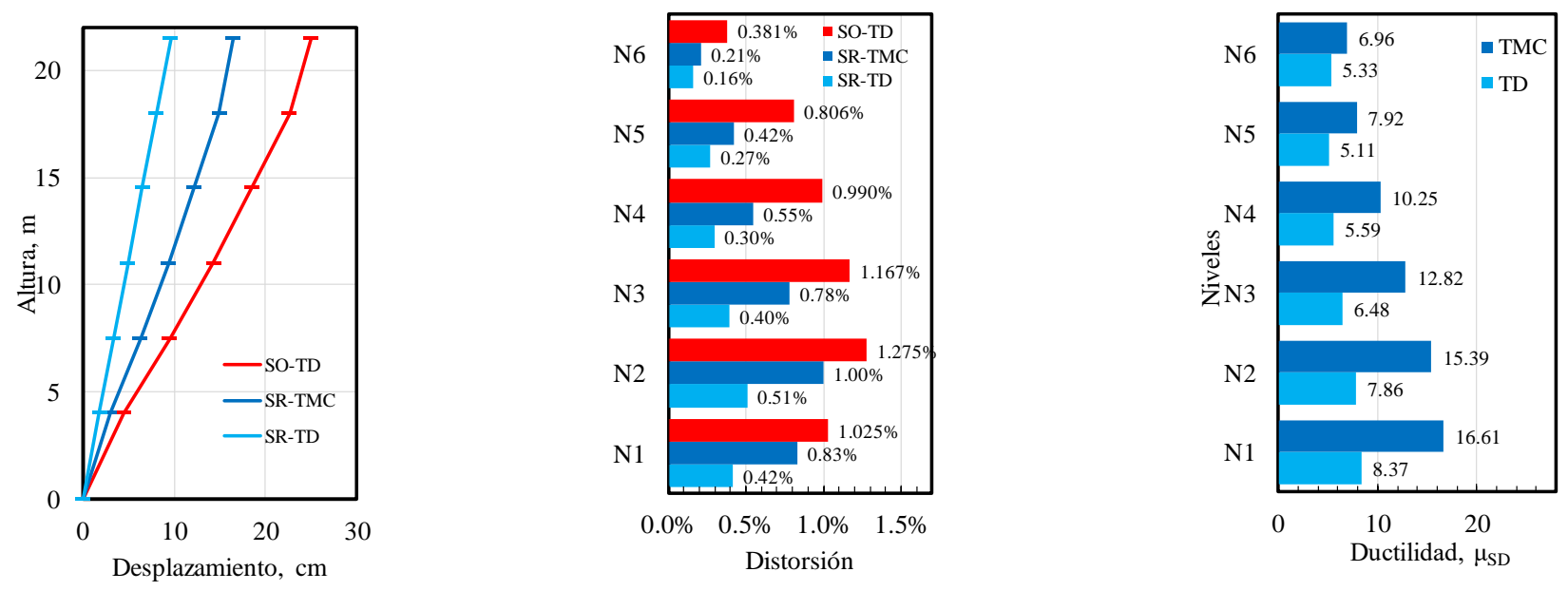

Figura 3 Demandas de ductilidad del SD promedios para registros TD y TMC.

\section{Conclusiones}

En este trabajo se presentó la aplicación de un método de diseño propuesto para el refuerzo de estructuras existentes. El método se basa en los criterios del diseño por desplazamiento y permite considerar dos niveles de demanda sísmica. Parte de un análisis estático no lineal para caracterizar la estructura existente, y luego, mediante el concepto de amortiguamiento viscoso equivalente de sistemas en paralelo determina la cantidad de resistencia y rigidez a incorporar según el desplazamiento objetivo. Las propiedades del sistema de disipación se distribuyen entre los dispositivos de los distintos niveles, manteniendo la forma de vibrar del primer modo de la estructura original.

La confiabilidad del método aplicado se mostró mediante la aplicación del mismo al refuerzo de un edificio de hormigón armado de 6 niveles. El edificio se sometió a 4 grupos de 7 registros sísmicos. Encontrando que la diferencia entre el desplazamiento propuesto en el diseño y el obtenido del ADNL no superó el 10\%; mientras que las demandas de ductilidad del ADNL resultaron inferiores a las supuestas en el diseño. Por lo tanto, se ha conseguido un método de diseño del sistema de disipación para reforzar estructuras que cumple con los objetivos propuestos.

\section{Agradecimiento}

El autor agradece a la Universidad Tecnológica Nacional, por el financiamiento económico para esta investigación, a través del Programa de Becas Doctorales y de los proyectos ECIFNME0004491 y ECUTNSR 6616. En particular al GEDEC y a la Regional San Rafael, lugar donde se desarrolla la investigación.

\section{Referencias}

Abrahamson, N., (1992). “Non-stationary spectral matching”. Seismological Research Letters, 63(1), p. 30. ASCE 41, (2017). "Seismic Evaluation and Retrofit of Existing Buildings, USA": American Society of Civil Engineers. ASCE 7, (2016). "Minimum design loads for buildings and other structures", Reston, Virginia, EEUU: American Society of Civil Engineers.

Bay, C. y Palazzo, G., (2019). “Amortiguamiento Viscoso Equivalente en el Diseño Basado en Desplazamientos”. Revista Tecnología Y Ciencia, Volumen 36, pp. 115-129. 
Bay, C., Palazzo, G. y Prados, J., (2019). “Diseño basado en desplazamiento para disipadores de energía empleado en refuerzo de edificios escolares". Mecánica Computacional XXXVII, pp. 129-138.

Bergami, A. y Nutti, C., (2013). "Design of dissipative braces for an existing strategic building with a pushover based procedure". Earthquakes and Structures, Vol. 4, No. 1 (2013) 85-108

ETABS, (2015). “Integrated Building Design Software Version 18”. Computers and Structures CSI.

Fajfar, P., (2000). "A nonlinear analysis method for performance based seismic design". Earthquake Spectra, p. 16(3):573-592.

FEMA-P695, (2009). "Quantification of building seismic performance factors, Washington": Federal Emergency Management Agency Publication.

INPRES-CIRSOC 103-I, (2013). "Reglamento argentino para construcciones sismorresistente. Construcciones en general”. Instituto Nacional de Tecnología Industrial. Bs. As.: INTI.

Kim, J.y Choi, H., (2004). “Behavior and design of structures with buckling-restrained braces”. Eng. Struct., pp. 26(6): 693-706.

Liu, T., Zordan, T., Briseghella, B. y Zhang, Q., (2014). "An improved equivalent linear model of seismic isolation system with bilinear behavior". Engineering Structures, pp. 61, 113-126.

Mazza , F. y Vulcano, F., (2015). "Displacement-based design procedure of damped braces for the seismic retrofitting of r.c. framed buildings. Bulletin Earthquake Engineering, p. (13):2121-2143.

Mazza, F. y Vulcano, A., (2008). "Displacement-based seismic design procedure for framed buildings with dissipative braces. (a) Part I: Theoretical formulation; (b) Part II: Numerical results". USA, American Institute of Physics conference proceedings, p. Part Two.

Nuzzo, I., Losanno, D. y Caterino, N., (2019). "Seismic design and retrofit of frame structures with hysteretic dampers: a simplified displacement based procedure”. Bulletin of Earthquake Engineering, Volumen 17, p. 2787-2819.

Ponzo, F.C.; Dolce, M; Vigoriti, G.; Arleo, G.; Di Cesare, A.; (2009). "Progettazione di controventi dissipativi a comportamento dipendente dagli spostamenti". Bologna, Italia, s.n.

Priestley, M. y Grant, D., (2005). "Viscous damping in seismic design and analysis". Journal of Earthquake Engineering, pp. 9:sup2, 229-255.

Priestley, M., Kowalsky, M. y Calvi, M., (2007). Displacement based seismic design of structure. Pavia, Italia(Pavia): IUSS Press.

Yan, L., Gon, J. y Zhang, Q., (2018). "Investigation of Global Equivalent Damping and Statistical Relationship of Displacement Between Nonlinear Static and Dynamic Analysis of Reinforced Concrete Frame Structures". Earthquake Spectra, pp. (34):1311-1338. 